\section{$\underset{\substack{\text { hommes } \\ \text { \& migrations }}}{ }$}

\section{Hommes \& migrations}

Revue française de référence sur les dynamiques

migratoires

$1295 \mid 2012$

Algérie - France : une communauté de destin

\title{
L'histoire et la position de la victime
}

\section{Benjamin Stora}

\section{(2) OpenEdition \\ Journals}

\section{Édition électronique}

URL : http://journals.openedition.org/hommesmigrations/1075

DOI : 10.4000/hommesmigrations. 1075

ISSN : 2262-3353

\section{Éditeur}

Musée national de l'histoire de l'immigration

\section{Édition imprimée}

Date de publication : 1 janvier 2012

Pagination : 110-118

ISSN : 1142-852X

\section{Référence électronique}

Benjamin Stora, «L'histoire et la position de la victime », Hommes \& migrations [En ligne], 1295 | 2012, mis en ligne le 01 janvier 2014, consulté le 10 décembre 2020. URL : http://journals.openedition.org/ hommesmigrations/1075; DOI : https://doi.org/10.4000/hommesmigrations.1075 


\section{L'histoire et la position de la victime}

Par Benjamin Stora, professeur des universités à Paris-13 ${ }^{(1)}$

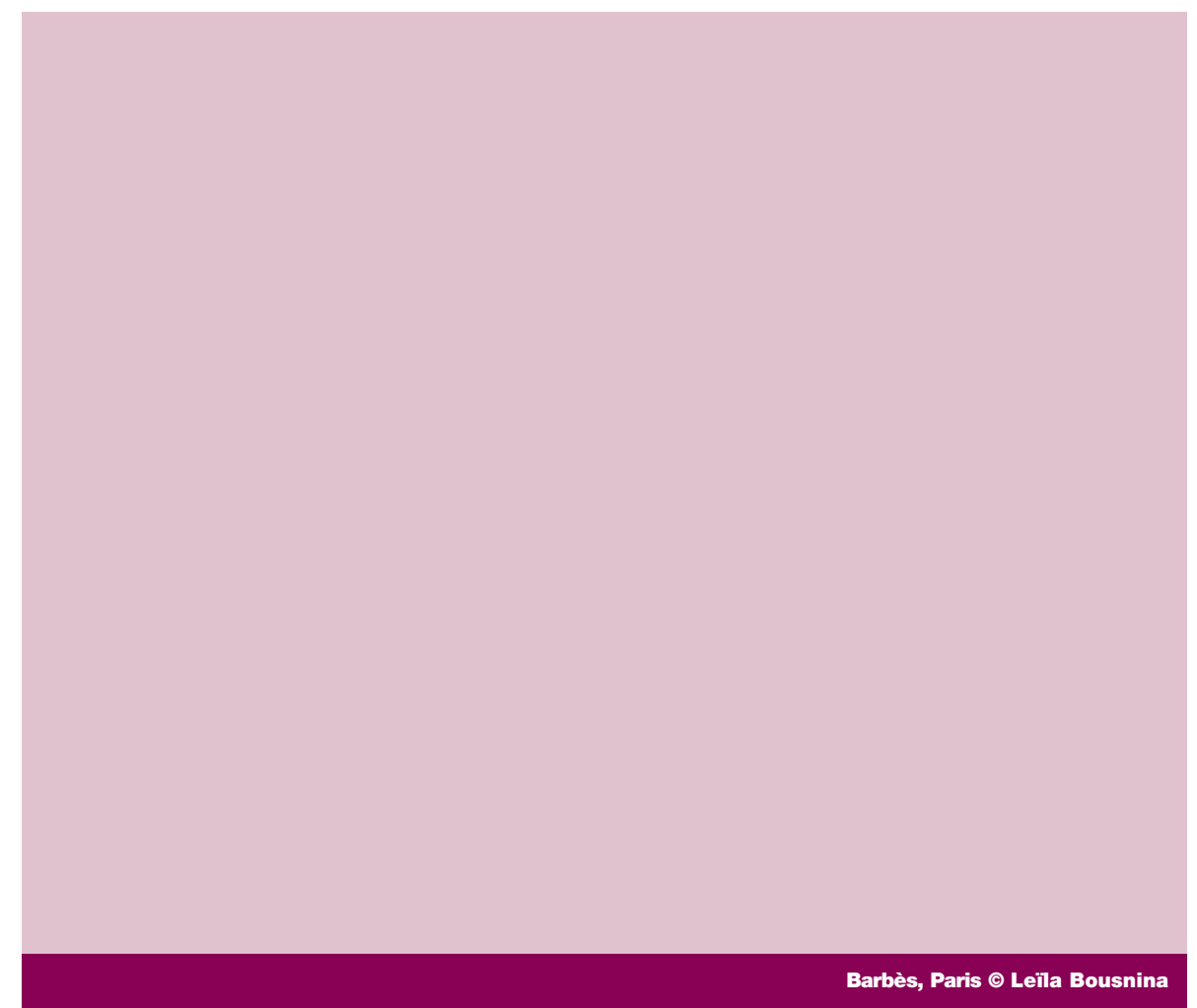

Depuis une quinzaine d'années, en France, la concurrence des revendications mémorielles s'est développée sur les lacunes de

l'enseignement de l'histoire de la colonisation et de l'immigration.

Dans le cas de la France et de l'Algérie, la difficulté à revenir sur des épisodes historiques sombres détermine largement celle de penser un avenir commun dans un espace culturel mixte. II s'agit de dépasser une relation postcoloniale où la cacophonie des discours masque le développement réel des échanges entre les peuples. 
Le thème de la guerre des mémoires est monté en puissance dans la société française depuis une dizaine d'années. Il illustre une situation de crise, une difficulté à se projeter dans l'avenir. Le sentiment d'exacerbation mémorielle est très puissant car la mémoire est devenue une revendication à caractère politique émanant d'un certain nombre de groupes. Il accompagne le "communautarisme" à l'ceuvre dans la société française. Le thème s'enracine de manière politique avec une hiérarchisation des mémoires, des histoires particulières, des victimes et des bourreaux désignés.

Dans le même temps, l'histoire en tant que discipline est devenue une référence essentielle dans les représentations idéologiques. Dans les années soixante, la discipline en vogue dans les sciences humaines était la sociologie. Elle donnait le sentiment d'être en prise sur l'actualité et sur les transformations du présent, avec les grands mouvements d'engagement politique de l'époque. Aujourd'hui, l'histoire et la relation au passé conditionnent notre façon d'être dans le présent : l'histoire s'est installée au cceur de la vie intellectuelle française. Ce retour perpétuel du passé est certes enrichissant pour la connaissance des sociétés. Mais il renvoie aussi de manière évidente à une panne d'avenir. La recherche dans le passé de recettes rassurantes fabrique de la paresse intellectuelle et permet de se légitimer en permanence non pas comme acteur, mais comme victime dans l'histoire. Car, à la différence des années soixante-dix et quatre-vingt, la posture de victime est nettement privilégiée par rapport à celle d'acteur. Dans les décennies ultérieures, ce qui dominait, c'était la perception d'être soi-même un acteur de l'histoire et de pouvoir changer le monde. Ce qui prime aujourd'hui est le sentiment d'être une victime de l'État, des autres groupes de mémoire, des autres groupes sociaux. D'une certaine façon, l'histoire échappe ainsi à notre contrôle.

Ces trois raisons évidentes (la place éminente de l'histoire, la panne d'avenir et la posture victimaire) renvoient à des crises idéologiques très puissantes. Le discours dominant nous installe dans le passé, sous la forme d'une nation ancestrale s'inscrivant dans une longue durée très déterministe. Une certaine vision nationaliste du monde crée un "nous" et un "eux", une histoire ancienne qui est la nôtre et une histoire nouvelle qui est la leur. Chaque groupe de "nouveaux" arrivants entre en concurrence avec les autres groupes, ce qui renforce le risque de dilution nationale et de conflits. Face à ces mouvements réels, la France prend un certain retard, dû à la réticence à vouloir affronter l'histoire, notamment l'histoire coloniale dont elle a voulu se débarrasser rapidement après l'indépendance de l'Algérie. Nous payons maintenant près de quarante années de mise à l'écart de cette histoire, ce qui se traduit par une "guerre des mémoires". Le "rattrapage" s'effectue de différentes manières : par le travail d'une nouvelle génération de chercheurs et aussi, de manière plus contestable, par l'intervention de l'État. 


\section{Les demandes mémorielles}

Pendant longtemps, l'État n'est pas intervenu dans les questions d'histoire. Il a commencé à le faire en 1990, à la suite des revendications portées par des responsables de la communauté juive qui se sont battus dans les années soixante pour la reconnaissance officielle de la négation de la responsabilité de Vichy dans la déportation des juifs. Les grandes étapes sont connues, à commencer par la lutte de Serge Klarsfeld et des associations des fils et petits-fils de déportés puis, en 1987, avec le procès Barbie, et finalement par le vote de la loi Gayssot en 1990. À cette époque, une mobilisation importante de groupes porteurs de mémoire a contraint l'État à assumer ses responsabilités sur le plan historique et mémoriel. Ces combats ont encouragé d'autres groupes de mémoire à faire valoir à leur tour leurs droits. Dans cette brèche ouverte se sont inscrits les mouvements arméniens, la mobilisation autour de la question noire avec la loi Taubira de 2001, et les mouvements des piedsnoirs et des harkis qui se sont présentés comme des victimes abandonnées par la France, et non comme des acteurs de l'histoire coloniale.

Lintervention de l'État s'est donc manifestée avec la loi Gayssot de 1990, le discours de Chirac sur Vichy en 1995, la loi Taubira de 2001, la loi sur le rôle positif de la colonisation de 2005 puis, plus récemment, avec la loi sur le génocide arménien. Face à cet investissement progressif de l'État s'est produite une double réaction : une, positive, de certains chercheurs qui ont estimé que l'État était enfin prêt à assumer ses responsabilités sur des réparations possibles, et une seconde, plus négative, d'autres chercheurs qui ont dénoncé un interventionnisme excessif de ce même État, qui devrait cesser de répondre aux sollicitations mémorielles (l'histoire ne peut pas s'écrire au Parlement).

\section{Le rôle de l’éducation}

Une autre question porte sur le rôle de l'État en terme d'éducation des jeunes générations. L'État a apporté à ces questions une réponse à travers des dispositifs législatifs, mais l'on peut se demander s'il n'aurait pas pu intervenir de manière différente, en mettant l'accent sur la culture et l'apprentissage de la citoyenneté. Des progrès ont néanmoins été accomplis, car certains manuels scolaires parlent aujourd'hui de la guerre d'Algérie et de la décolonisation. Les années quatre-vingtdix ont marqué un tournant dans la fabrication de ce savoir. Mais le retard était si important que les populations issues des anciennes colonies ont, aujourd'hui encore, un sentiment d'impuissance et de vide au regard du traitement de ces questions à 
l'école. Ensuite, les enseignants sont peu préparés à aborder ces questions dans leurs classes. S'il faut partir du sommet des universités pour élaborer et enseigner un savoir qui doit se diffuser ensuite dans l'ensemble de la société, force est de constater que la France a pris un retard considérable dans ce domaine. Le premier colloque officiel sur la guerre d'Algérie, organisé par Jean-Pierre Rioux à l'Institut du monde arabe avec le soutien du ministère de l'Éducation nationale sur le thème "Apprendre/enseigner la guerre d'Algérie", s'est tenu en 2001.

Les universités françaises manquent d'une approche transdisciplinaire qui permettrait d'aborder la langue, l'histoire, la littérature,

Une tendance majoritaire souhaite ne pas toucher au statu quo ancien et refuse de regarder les groupes minoritaires qui se battent : c'est le discours dominant de l'anti-repentance. la civilisation, donc de fédérer les chercheurs autour d'un même thème. Le traitement des questions historiques et mémorielles est laissé à l'intérêt ou à l'initiative personnels. Alors que l'Éducation nationale marque le pas et cherche ses marques, le cinéma a construit un ensemble d'imaginaires où le thème de l'histoire et de la mémoire coloniale et postcoloniale est abordé : entre 2005 et 2010, sept films ont pris pour sujet la guerre d'Algérie (La Trahison, Nuit noire, Harkis, Cartouches gauloises, Mon colonel, L'Ennemi intime, Hors-la-loi), ce qui est tout à fait considérable.

\section{La sortie du silence?}

Si l'on considère les célébrations officielles, les commémorations à l'initiative de l'État, les “incursions" de l'Éducation nationale et les cuvres cinématographiques, on voit bien que les efforts existent, alors même que persiste le sentiment que tout est précaire, fragile, censuré, caché... La sortie du silence, de la "La Gangrène et l'Oubli(2)", est effective. Mais cette sortie s'effectue dans le désordre, sans concertation, réflexion, projet, mise en débat dans la société. Dans une sorte d'“anarchie" et de foisonnement. La sortie du silence a débouché sur une guerre des mémoires. Dans ce contexte, la volonté de créer des passerelles pour comprendre la douleur des autres groupes porteurs de mémoire est faible. Au contraire, domine le désir de cacher les souffrances des autres, au profit de son propre passé et de sa propre souffrance. Il ne s'agit pas de briser les tabous pour que toutes les souffrances soient reconnues, mais bien au contraire de marquer que certaines souffrances sont exclusives. 
Cette sortie de la censure se fait dans un contexte où des parties importantes de la société française regrettent le silence. Une tendance majoritaire souhaite ne pas toucher au statu quo et refuse de regarder les groupes minoritaires qui se battent : c'est le discours dominant de l'anti-repentance. Les nombreux pamphlets récents sur ce thème ont été de véritables succès, de même que le discours de Nicolas Sarkozy pendant sa campagne présidentielle de 2007 : "Nous ne voulons plus que l'on parle de cette histoire." La sortie du silence n'est pas toujours acceptée. Par exemple, les nombreux films sur le thème de la guerre d'Algérie ont été des échecs relatifs du point de vue de leur audience. Les films racontent les événements dans leur complexité, mais l'autocensure dans le public atteste le refus de rentrer dans cette histoire traumatique.

Laccent est majoritairement mis sur le fait qu'il faut éviter de revenir sur l'histoire passée pour se projeter dans l'avenir. Et le rapport au passé reste très compliqué. Il est difficile de se situer à la bonne distance entre la glorification du passé colonial de la France et la critique de ce même passé qui a uniformisé et détruit d'autres cultures.

\section{La place et le rôle de l'histoire de l'immigration}

Dans les années quatre-vingt, sortaient les premières études sociologiques et historiques sur l'immigration en France, ainsi que les premiers grands ouvrages tels que Le Creuset français de Gérard Noiriel ${ }^{(3)}$. Mais beaucoup de grands historiens ne disaient presque rien sur l'immigration maghrébine et algérienne en France, alors qu'il s'agit d'un phénomène très ancien qui remonte aux années vingt. À cette époque, ce sont les travaux d'Abdelmalek Sayad qui ont ouvert la voie. En 1990, à l'Arche de la Défense, s'ouvrait la première grande exposition, organisée par Driss El Yazami sous ma direction scientifique, sur le thème "France des étrangers, France des immigrés".

Le champ académique français est marqué par un retard de l'histoire de l'immigration, perçue comme une histoire séparée de la France qui s'est développée selon un modèle de matrice jacobine centralisée. Ensuite, à l'intérieur du champ des études migratoires, l'histoire des migrations coloniales et postcoloniales s'est développée après l'histoire des migrations européennes, alors que les "témoins" des migrations coloniales étaient présents en métropole à la même époque que les Italiens, les Polonais et les Espagnols, dès les années trente. Cependant, à cette époque, il fallait "oublier" l'histoire coloniale, et plus particulièrement celle des immigrés coloniaux eux-mêmes. 
Lorsque l'histoire coloniale a surgi dans le débat public dans les années quatrevingt-dix, on a commencé à parler des "immigrés coloniaux". Ces derniers subissent donc une double mise en périphérie dans le champ des études historiques : d'une part, une mise à l'écart propre à chaque immigré et, d'autre part, une mise à l'écart au sein même des études migratoires. Si on regarde l'histoire des migrants italiens dans l'est de la France, en Lorraine par exemple, il faut savoir qu'à la même époque de nombreux immigrés algériens travaillaient dans les usines de sidérurgie, dans une situation de grande précarité, aggravée par des luttes internes et des règlements de comptes violents, sans que cela soit reporté dans les études d'histoire. Cette double mise à l'écart s'explique par le fait que ces immigrés se sont assumés comme tels dans les années soixante-dix et quatre-vingt seulement, lorsqu'ils ont décidé de rester en France : le champ des études académiques s'est alors ouvert à eux.

\section{Renouveler la pensée de l'expérience migratoire}

Les générations issues de l'immigration se sont elles aussi intéressées à leurs parcours. L'émergence d'une génération à partir des mouvements citoyens des années quatrevingt a commencé à investir le champ des études académiques tout au long des années quatre-vingt-dix. Il s'agit, certes, d'un transfert intergénérationnel, mais la question du retour disparaît alors des études migratoires. L'histoire de l'immigration en France souligne l'existence des migrations temporaires. Par exemple, l'étude de l'histoire des migrations saisonnières est très faible, car l'immigration est perçue uniquement dans une perspective de long terme et d'intégration. Mais cela n'a pas été toujours le cas. Auparavant, le modèle central de la figure immigrée était celui du migrant qui repart dans son pays après un séjour plus ou moins long. En revanche, l'histoire de l'émigration, du point de vue des pays d'origine, est très peu étudiée. On considère que c'est aux chercheurs des pays d'origine de mener ces recherches, et qu'il y a de manière inconsciente une sorte de séparation des tâches. Les chercheurs français s'interrogent peu sur les raisons du départ. Dans ma trajectoire intellectuelle, j'ai commencé à travailler sur les sociétés de départ, au Maghreb, pour ensuite m'interroger sur l'immigration en France. Cette méconnaissance des sociétés de départ est préjudiciable à l'étude des migrations en France.

La distinction est forte entre pays de départ et société d'arrivée ; de la même manière, les recherches distinguent l'immigration économique d'un côté et celle, politique et familiale, de l'autre. Or, dans un souci louable de classer et de catégoriser les phénomènes, il faut toujours garder à l'esprit qu'un homme ou une femme part avec des 
désirs de liberté face à des sociétés de départ problématiques, et non pas seulement pour des raisons économiques. L'immigration est également liée à l'absence de libertés dans le pays d'origine et à l'attirance pour l'Europe où l'on sait, grâce aux nouvelles techniques de communication et d'information, que l'on peut vivre dans des espaces citoyens libres dans lesquels les droits sont respectés. D'ailleurs, le départ de la jeunesse constitue une sanction des sociétés du Sud. "Pourquoi les jeunes ontils comme seul horizon la fuite ?" C'est une question liée à la crise de la décolonisation et, plus particulièrement, à la crise des équipes dirigeantes de ces pays depuis cinquante ans. Dans le sillage des études sur les migrations, se sont développées les études sur la colonisation.

\section{L'essor récent des études postcoloniales}

Une forte poussée de jeunes chercheurs sur l'histoire coloniale se manifeste aujourd'hui en France. S'ils veulent travailler sur ce thème, le nombre de professeurs capables de diriger leurs recherches reste néanmoins très réduit. On pourrait dire que l'on paye "physiquement" ce retard dans la recherche, dû au fait que les pouvoirs publics se sont désinvestis de l'histoire coloniale dans les années soixante à quatre-vingt-dix. Par ailleurs, il faut définir ce qu'est le postcolonial. Est-ce faire l'histoire de la colonisation? travailler sur les traces de l'histoire de la colonisation dans les sociétés postcoloniales ? Ou bien est-ce tout ce qui relève de l'écriture littéraire, comme dans les sociétés anglo-saxonnes, où l'histoire est hybridée à travers la littérature?

En France, le postcolonial a tout simplement à voir avec l'histoire coloniale, et non pas à ce rapport avec la langue. Cette idée de penser au colonial et au postcolonial à travers la langue et la littérature est très récente en France. Elle émerge depuis une quinzaine d'années à travers ce que l'on appelle la "francophonie". Mais n'appelle-ton pas "francophonie" ce "postcolonial" que l'on voudrait gommer ? Or la francophonie apparaît comme un espace linguistique et culturel issu d'un postcolonialisme ambigu. En France, cette posture n'est pas assumée, alors qu'au Royaume-Uni elle est clairement affirmée, dans le rapport aux écritures indiennes par exemple. En France, la littérature d'expression française n'est pas reconnue comme une littérature d'anciens coloniaux, mais comme une simple littérature francophone. La référence à l'histoire de l'empire est niée.

La France veut être considérée comme une "grande nation", sans assumer que cette grandeur lui venait de son ancien empire colonial. Le général de Gaulle avait 
compris que la France n'existait pas au regard des puissances internationales sans son empire. Il a eu cette intelligence politique en 1940, en résistant avec l'appui des colonies, puis en 1944 avec la conférence de Brazzaville. Il affirmait que la France n'était pas seulement la France métropolitaine, mais la "Grande France" de l'empire colonial. Sa conception du nationalisme français était fondée sur l'expansionnisme colonial. Il est vrai que, grâce à l'armée d'Afrique, après 1944, la France redevient une grande puissance capable de s'asseoir à la table des "grands".

Les principaux écrivains français connus à l'étranger sont originaires de cet espace : Patrick Chamoiseau, Assia Djebar, Kateb Ya-

La France veut être
considérée comme
une "grande nation",
sans assumer que
cette grandeur lui venait
de son ancien empire
colonial.

cine... Ils sont connus comme des écrivains de langue française venus d'une histoire complexe, aujourd'hui à la fois périphérique et centrale dans l'imaginaire français. De nos jours, il serait impensable d'écrire sur les lieux de mémoire de la France sans parler des lieux liés à la colonisation et à la décolonisation, comme Diên Biên Phû, alors qu'il s'agit de lieux hautement problématiques liés à des défaites, synonymes de perte, de retrait, d'abandon.

\section{Entre la France et l'Algérie}

Entre ces deux pays, nous sommes restés au stade de la recherche d'un simple partenariat économique. La vision stratégique politique ou culturelle ne s'est jamais vraiment construite. Au contraire, un durcissement des discours est apparu des deux côtés de la Méditerranée. Les échanges culturels et les flux migratoires existent historiquement, mais les États se positionnent plutôt dans une sorte de décalage historique. L'immigration algérienne en France est un phénomène très ancien, avec la présence d'une quatrième génération de Français originaires de cette immigration : cela crée une sorte d'espace culturel et social mixte, extraordinaire pour les deux pays. À eux seuls, les Algériens présents en France et les Français d'origine algérienne incarnent sur le plan physique un espace spécifique qui fonctionne selon des codes mémoriels et sociaux communs aux deux pays. Pourtant, de part et d'autre de la Méditerranée, les États restent dans une posture nationaliste "à l'ancienne", fondée sur des conceptions archaïques du nationalisme, qui produit de nouvelles fabrications idéologiques : en Algérie avec la glorification 
de la guerre d'indépendance, et en France avec le discours de l'anti-repentance. Or ces espaces culturels mixtes existent, les étudiants et les chercheurs circulent d'une rive à l'autre, de même que les familles. Il serait impensable d'arrêter cette circulation de personnes appartenant aux deux rives. Il s'agit d'un monde réel, mais qui reste virtuel sur le plan politique. Il faut donc avancer sur la traduction politique de cet espace culturel mixte, et ne pas y voir uniquement une réalisation au seul bénéfice de l'économie.

Ce mouvement s'élargira de manière naturelle, car trop d'hommes et de femmes en France et en Algérie appartiennent aux deux pays, ont tissé des liens dans l'un comme dans l'autre. Certes, l'immigration algérienne ira partout où l'on pourra l'accueillir, mais il n'y a qu'avec la France que deux ou trois millions d'hommes et femmes d'origine algérienne ont une histoire commune. Cet espace social et culturel mixte ne pourra pas être défait, car il est désormais enraciné dans les deux pays : le nombre de binationaux vivant en France est considérable, plusieurs centaines de milliers de personnes. La réunion des entrepreneurs économiques, culturels et artistiques, et l'instauration d'un espace culturel permettent d'inverser la tendance d'une hégémonie économique.

Rien n'empêche les intellectuels de se fixer cet objectif de travail, mais les contraintes matérielles sont fortes, et il ne faut pas oublier les rivalités politiques du côté sud de la Méditerranée. L'affrontement entre l'Algérie et le Maroc constitue en effet un frein considérable au développement d'une union méditerranéenne. Ces replis nationalistes sont une entrave. Comment prétendre avoir un dialogue équilibré avec la rive nord sans circulations transversales entre les pays du Sud sur le plan culturel ? C'est aux intellectuels du sud de la Méditerranée de poser les bases de ce futur dialogue culturel, en s'appuyant sur le puissant mouvement démocratique qui traverse le monde arabe aujourd'hui. De son côté, la France risque de mettre à l'écart son héritage culturel et mémoriel du Sud, pour se jeter dans la mondialisation. Or il est très dangereux d'essayer de se débarrasser de l'histoire, car celle-ci revient toujours violemment.

\section{Notes}

1. Dernier ouvrage paru : La Guerre d'Algérie expliquée à tous, Paris, Seuil, 2012. www.univ-paris13.fr/benjaminstora/

2. Benjamin Stora, La Gangrène et l'Oubli. La mémoire de la guerre d'Algérie, Paris, La Découverte, 1992.

3. Coordonné par Yves Lequin et édité en 1987 chez Larousse. 